\title{
Tables of event sequences for sequential analyses of data in psychological experiments containing two-class events ${ }^{1}$
}

WILLIAM E. VANDAMENT, RICHARD G. BURRIGHT, ROLLIN R. FESSENDEN, and WILLIAM $\mathrm{H}$. BARKER, ${ }^{2}$ STATE UNIVERSITY OF NEW YORK AT BINGHAMTON, Binghamton, New York 13901

Tables of sequences of two-class events are presented for use in programming psychological experiments in which behavior on trial $n$ may be a function of the events of trials $n-1, n-2$, and/or $n-3$. Various factors related to schedule generation are discussed, i.e., restrictions on trial-block length which accompany sequential balance, interrelationships of trial blocks in the multiblock experiment, relationships between run length and $r$-tuple occurrences, and alternation behavior. Following a consideration of various methods of schedule generation for the two-class experiment, it was concluded that no method can result in schedules that possess all properties considered desirable in psychological experiments. However, the present sequences allow for sequential balance and analysis, and thus should prove useful in producing schedules in some contexts that are standard with regard to sequential influences.

The following tables contain computer-generated sequences characterized by equal numbers of two-class events (designated as 1 or 0 ), which can be utilized in programming psychological experiments involving several presentations of each event when sequential analyses of behavior and counterbalancing of event sequences are desired. Although sequential analyses have already been used to define much about learning processes which could not be observed directly from the customary trial-block summary measures, these tables constitute the first presentation of a variety of schedules for which transitional occurrences $(11,10,01,00)$ are balanced within trial blocks for various numbers of trials preceding the measurement trial.

\section{APPROACHES TO EVENT SEQUENCES}

The sequential arrangements of treatments in experiments have long been of concern to psychological investigators. Random methods of event generation, of course, have been utilized in many experimental contexts to minimize various artifacts related to average placement and pattern discriminability of treatments. However, without restrictions the random approach, by definition, disallows any control of various aspects of sequential occurrences within a finite experimental situation. As will be discussed later, to obtain certain types of control, the nature of restrictions on randomization can become quite complex.

Gellermann (1933) constructed a set of 44 series in an attempt to cope with problems in discrimination learning related to position preferences and tendencies of Ss to alternate responses in experiments containing two response alternatives. Each of the Gellermann series contains 10 events, 5 of each type, with the possibility existing for series to be combined to provide for the programming of an experiment length that is some multiple of 10 .

Some investigators (cf. Frey, 1969; Moore, 1964) have utilized schedules composed of an equal number of runs of lengths 1,2 , and 3 for each of two event classes. These schedules have lengths that are some multiple of 12 events, e.g., the sequence 101100111000 has the specified properties. When each sequence serves as one of a series of trial blocks and when a transition from one event class to the other class occurs at each junction between successive blocks, these schedules are characterized by each event class having been preceded equally often by events of both classes.
Various Latin-square designs have been developed to deal with somewhat different concerns about the sequential patterns of treatment presentation (e.g., Bradley, 1958; Cochran \& Cox, 1957; Grant, 1948; Patterson, 1952). These designs typically have involved rather short series of events and three or four different types of treatments. Additionally, these designs usually cover situations in which treatments are not repeated for the same individual, or are repeated only once. The basic concerns in their development have been to ensure that each treatment has the same average placement in the sequence and that each treatment has been preceded equally often by every other treatment.

In the development of the present sequences, the experiments of primary concern have been those involving two-class events with several, but equal, repetitions of each event, e.g., experiments in differential conditioning, discrimination learning, partial reinforcement, and probability learning. The goal has been to develop sequences in which the residual effects of trials $n-1, n-2$, and/or $n-3$ could be balanced and statistically assessed for individual Ss. Thus, although all of the listed methods are related to each other in some respects, none share identical properties, and each has been developed to deal with different situations or concerns.

\section{PROPERTIES AND GENERATION} METHOD OF PRESENT SEQUENCES

Conceptually, each of the present series TABLE 1

TERMINAL EVENT OF PRECEDING SEQUENCE : 1

$\begin{array}{ll}\text { 1. } 0011 & 2.1001\end{array}$

TABLE 2

SEQUENCE LENGTH $=8$ SUBSEQUENCE LENGTH: $=2$

TERMINAL EVENT DF PRECEDING SE QUENCE : 1

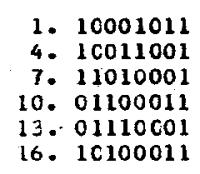

2. 10001101
5. 11001001
8. 00011011
11. 00111001
14. 10010011
17.00011101
3. 00010111
6. 00110011
9. 01000111
12. 11000101
15. 00100111
18. 10110001 
TABLE 3

SEQUENCE LENGTH $=8$

TERMINAL EVENTS UF

PRECEDING SEQUENCE : 11
SUBSEQUENCE LENGTH $=3$

TERHINAL EVEMTS CF

PRECEDING SEOUENCE : 10

$\begin{array}{ll}\text { 1. } 00010111 & \text { 1. } 11100010 \\ \text { 2. } 11000111 & 2.00101110 \\ \text { 3. } 10100011 & 3.00111010 \\ \text { 4. } 10001011 & 4.10001110\end{array}$

TABLE 4

S EOUENCE LENGTH $=12$ once by 0 , behavior can be analyzed as a function of the event immediately preceding the measurement trial.

In Table 2 each of the eight-event sequences contains two each of the possible 2-tuples, if the terminal event of a preceding sequence is counted. The eight-event sequences of Table 3 are a subset of those in Table 2 which contain all possible 3-tuples, i.e., 111, 110, 101, 100, $011,010,001$, and 000 . For these Table 3 sequences, each class of event is preceded equally often by each 2-tuple, so that it is possible to assess the effects of events of trials $n-1$ and $n-2$ on behavior occurring at trial $\mathrm{n}$.

Table 4 shows sequences of length 12 having equal representation of all length-2 subsequences, and Tables 5 and 6 list 16-event sequences balanced for 3 - and 4-tuple frequencies, respectively. Thus, due to the 4-tuple balance in Table 6, each event class has been preceded by all 3-tuples, so that the effects of events on trials $n-1, n-2$, and $n-3$ can be assessed. In general, this type of assessment can be performed for the $I-1$ trials preceding the nth trial whenever r-tuple balance is achieved.

The programming logic employed in the generation of these sequences involved the systematic production of all possible sequences of the specified length. Following the generation of a sequence, an assessment was made for the appropriate number of $1 \mathrm{~s}$ and $0 \mathrm{~s}$, and the numbers of $r$-tuples or overlapping subsequences of length $r$. The iefore, the tables should be exhaustive with regard to possible sets for the relevant characteristics in each case. (Note, however, Point 4 below regarding complements or mirror images of the tabled sequences.)

Various other factors governing use of the tables also deserve comment:

(1) Each r-tuple can be represented as a branch in a Markov diagram and each event in the trial block as the final event of such a branch. Therefore, the basic length of each trial block (i.e., prior to the addition of any "terminal events" which precede it) is related to some multiple of the number of branches that exist for a given subsequence length. Thus, for 2-tuples there are $2^{2}$, or 4 , possible branches and the basic length of a balanced trial block must be some multiple of 4 , e.g., $4,8,12$, 16,20 , etc. For schedules balanced for 3-tuple occurrences, the basic trial block length is restricted to a multiple of $2^{3}$, or 8 , while for length-4 subsequence balance, the basic trial block length must be a multiple of $2^{4}$, or 16 .

(2) When several sequences are used in succession, the initial event(s) of a given sequence is/are normally the final event(s) 
1. $1100101000 C 11011$

3. 0100011110100011

5. Ccosc1111 CCC1011

7. 0000100111161011

s. 1000110100010112

11. 0011000010101111

13. CC10000101110111

15. CC11101C1CCCCIII

17. $101101000 \mathrm{ClCCl11}$

15. 101100001CC10111

21. 1100100001161011

23. $011101001 \mathrm{cec} 111$

25. 1010100110000111

27. $110010110 \operatorname{coc} 1011$

29. 010000140111011

31. 1001000010110111

33. 1000010106111011

35. $100001001 C 110111$

37. CCO011001C1C1111

39. C10011110CCC1011

41. CO100001011C1111

43. Co10101i1cocc111

45. $110100010 \mathrm{CO} 11011$

47. 1100010001101011

49. $11011000 \mathrm{CICC1OL1}$

1. 10C101C11CACO111

53. $1010100011 \mathrm{COL1}$

55. $110000101 C 110611$

57. COJO11C10C1C1111

5s. Cous11101C1coull

e1. 110101 Col1ccoll

63. C110C100OC1C1111

65. $100001611 C 01 C 111$

67. 0000101011160111

69. 1010000111001011

71. 1001010000111011

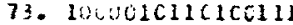

73. Clolouvililcuoll

77. 1luxclicicicall

7s. COL101000C1C1111

E1. li., J161C1:1Ccon

E3. $00001011110 \mathrm{C} 1011$

85. $100 \mathrm{Col11}$ C $1 \mathrm{C1011}$

87. 1010100001110011

89. C0001011010c1111

\$1. 1001101000010111

53. C1C111 COL COCOLll

55. C11110CO1 COC1011

57. 1100c11c1Coc1011

99. 1000010111010011

101. C11100 C1CCO1C111

103. C00010111C010111

1C5. C010100001111011

1C7. 100101C11106001L

1C9. 1100010101160011

111. C0010001111C1011

113. 1011 ccolocolol11

115. $100001101 \mathrm{C010111}$

117. 1100Clellcec1011

119. 1011100100001011

121. 0001000110151111

123. 1100100001011011

125. 11CC1011010C0011

127. CC1L1G100GC10111

129. 100311101 cocioll

131. CoulC1101 CCC1111

133. C110500101CC1111

135. CC1111C10COC1011

137. 1000011101001011

135. 1CCC1110101 Co011

141. $\operatorname{co0} 100101111011$

143. 011001011100C0111

145. Códiolco11c1111

147. 1011000010100111

149. 1610001000110111

151. CC1010110ccollil

153. 01016ccildct111

155. C10011600ClC1111

157. $(100001001111011$

155. 1101000011010011

161. 00011101010c0111

163. CCO1016uc 1111011
2. 0110010100001111

4. 0000100101110111

6. 010001110001011

B. 100010001011101

10. 1141001100001011

12. 0100010001111011

14. 0111100001001011

16. 000010111101001

18. 1000101000111011

20. 010010000111011

22. $0100100001111 \mathrm{cl}$

24. 1010011100001011

26. $1101010001100 \mathrm{Cl}$

28. 0100001011001111

30. 0110100100001111

32. 110100001301101

34. 010110601000111

36. 13001100010101111

38. 001011110100001

40. 101000100011101

42. 0100001110010111

44. 100001001011101

46. 4100011101000111

48. 1000100011101011

50. 1101000010110011

32. 101000010110011

54. 0100001011110011

56. 90001110100310111

58. 11 U1100100Julu1

60. 1000101011000111

b2. 000010011010111

64. 1000101110100011

06. 001010111100001

68. 1001101010003111

70. 000011010100111

72. 0010000101111011

74. 0110000100101111

76. 0011010100001111

73. 1000011010100111

80. 0100001100101111

¿2. 0101000011100111

84. 0101001110000111

86. 1100001101001011

5B. 1100001001101011

90. 1000010101110011

92. 000010102111011

94. 110110000101001

96. 00.11010001110111

98.1010110010000111

100. 1010011010000111

102. 110100101100001

104. 0000100101101111

106. 0001010111100011

103. 0100001011100111

110. 1010000110010111

112. 0102000011110011

114. O10011010uc01111

116. 1100001001011011

118. 1010001110001011

120. 011000100010111

122. 0101001111000011

124. 0001010001101111

126. $00010101100 \mathrm{Cl11}$

128. 010010000110111

130. 1010000110100111

132. 0100011000101111

134. $100010001 \mathrm{i} 01011$

136. 1101001000611011

138. 1000110101000111

140. 0001000111010111

142. 0011110101000011

144. 1010001011100011

146. 0111000011010111

148. 100101110000101

150. 110001011010001

152. 0010111100001011

154. $110.0110100001 \mathrm{Cl}$

156. 110001220101101

158. 110011010100001

160. 1100001010011011

162. 101 U501011C0011

164. 0000101100101111
165. Cou10001011C1111

167. 1011101001000011

169. 110001010 CO11011

171. 6000101110100111

173. $101001000 \mathrm{Cl11011}$

175. 1010100111000011

177. $0100111 \mathrm{ClCOCO111}$

179. Clu001101 c0c1111

181. $10101110001 \mathrm{C} 0011$

183. COOICI 10OCIC1111

1Es. C1C1COO1110CC111

1E7. $00101000011 C 1111$

189. 11001100001C1011

1S1. CO11100001010111

153. Clocuol1110c1011

195. $110101000 \mathrm{Cl1} 0011$

157. C111001ClCocol11

159. 1010111006010011

2C1. $11010001011 \mathrm{C0011}$

2C3. $010111100 \mathrm{CC} 10011$

2C5. $100010100 C 110111$

267. C00110100C1CI111

2C9. $11010011010 \mathrm{c0011}$

211. 101110001 CeC10.11

213. 1010011101000011

215. $100010111 \mathrm{COC1} 011$

217. $01001011110 \mathrm{C} 0011$

215. $101000010 \mathrm{Cl} 10111$

221. $1100101011 \mathrm{CCOO11}$

223. 1011100101000011

225. $011110001 \mathrm{ClCCO11}$

227. $10000100111 \mathrm{ClO11}$

229. C1111001010C0011

231. COJ100C101110111

233. C00111100C1C1011

235. C000111001010111

237. 0000111101010012

239. 11C10000110C1011

241. 1000100016110111

243. $101001000 C 110111$

245. 011010001 CCC1111

247. 1000010101160111

249. 0001000101111011

251. $101000111 \mathrm{ClC} C 011$

253. $110110100 \mathrm{CO} 10011$

255. C11110100C1c0011

257. $01011000010 c 111$

259. $101011000(16) 11$

261. $010100 \mathrm{C} 1 \mathrm{COC} 1111$

263. $000111010 \mathrm{co10111}$

265. $101110 \mathrm{COC} 10 \mathrm{C} 1011$

267. $100101000 C 110111$

269. $010001 \mathrm{Cl1COCl11}$

271. 101001011 COCC111

273. 1010010111000011

275. $101100101 \mathrm{coc} 011$

277. C00011110ClC1011

279. 110110001 COC1011

281. $10010111010 \mathrm{COCl1}$

283. C16010110c0C1111

285. 00C0111101CC1011

287. C1110000101C0111

289. $101110100 \mathrm{CO10011}$

251. 0000101001111011

293. $10000101110 \mathrm{Cl011}$

255. C1000100011c1111

297. $10101000111 \mathrm{co011}$

259. 01c11100001 c0111

301. C00110101COC1111

3C3. 1010011000010111

305. C000111C1ClCall

3C7. $11000 \mathrm{C1011001011}$

3C9. $110101100 C 100011$

311. 1010110001000111

313. Co1011101C0CO111

315. $11 \mathrm{J1} 01100 \mathrm{CO} 1001$

317. C10000111C1COL11

319. 1010000111010011

321. C1000101111c0011

323. $101100100 \mathrm{CC} 10111$

166. 0011110000101011

168. 1101101001000011

170. 1011100001010011

172. 1010000101110011

174. 1011010010000111

176. 0111010001000111

178. 1001110101000011

130. 110110100010001

102. 101006010011101

184. 000101110001011

186. 0101111001000011

$188.10100 \mathrm{C} 1100010111$

190. 0100111000010111

192. 1000011101010011

194. 100101101000011

196. 100010110100011

198. 0110001010001111

200. 001011010000111

202. 110000110010101

204. 0101001100001111

206. 0110100001001111

208. 1101000110100011

210. 0010110000101111

212. 0000100111010111

214. 1100011000101011

216. 0101100100001111

218. 101110001010001

220. 1010001101000112

222. 1001100001010111

224. 010000100110111

226. 1011101000100011

228. 0100010111000111

230. 1000110001010111

232. 011110100100001

234. 0010111000010111

236. 1000111000101011

238. 1010100011000111

240. 0010000111010111

242. 0100111101000011

244. 0000101011110011

246. 100111010000101

248. 0101111000100011

250. 100101100001011

252. 1101100010100011 
1. $1101111 \operatorname{coc} 1 \cos 0$

3. $01101111010 c 0010$

5. CC 1101001011110

7. cla111100co10110

9. c11C11110CUC1010

11. 1101001000011110

13. 1000011011110010

15. 1000011001111010

17. $101 C 00110 C C 11110$

19. 0010111011120010

21. CO1111010C010110

23. 100101111 COCO110

25. $\operatorname{coc} 101111 \mathrm{C1} 10010$

27. $10011110 \mathrm{CCO} C 110$

29. 101100001 col1110

21. Co1110100C111010

33. $10011 \mathrm{C} 100 \mathrm{CO} 11110$

35. 1000110100011110

37. $1110001 \mathrm{ClCOC1110}$

39. C10C001111C11010

41. 1100001001111010

43. 1110001110100010

45. Co101100C 1111010

47. 1111C11CC 10CCO10

49. $000101111 \mathrm{C1C0110}$

51. 110001111C0C1010

53. CC1011110C011010

55. 111000C1C 10C1110

57. 111100101cecollo

59. C1010000111C1110

t1. Co1101CoC1011110

Є3. GDCICO1J11110110

65 . COULOL1111C0110

57. $1160 \mathrm{Cl} 101111 \mathrm{CcOlO}$

69. $10001110001 C 1110$

71. 0C0111001C1C1110

73. $1110 C 0100 C 111010$

75. ColucollCIC11110

77. 1Crol111C11Coolo

79. $111001 \mathrm{clocc}(1110$

E1. CuUL11C111610010

E3. Co110101111c0010

E5. COLICLOLCCIIIIU

E7. $10011110110 \mathrm{CO010}$

89. $100111100 \mathrm{CC} 11010$

51. 0C01111011010010

53. $1000111 \mathrm{CLCC} 111 \mathrm{C}$

55. 100100C011011110

57. $1111010 C 011$ C0010

59. Co11C00101111010

101. $0100001101 \mathrm{cL111}$

1C3. 0010111000111010

1C5. 1100010001011110

1C7. 1C11100C211 c0010

1C5. 1111000011001010

111. C100001C11110110

113. CC1C00111C111010

115. 1CCOC1110C1C1110

117. Colo100011C11110

119. OCC1110101110010

121. $\cos 1 \mathrm{c} 1 \mathrm{cc} 11011110$

123. Co11CCO1111C1010

125. Cel101111CcClolo

127. 160001C011C11110

129. 001111ucol1c1010

1E1. 10C01ucellilol 10

123. Cuviol110C111010

135. 1110000111010010

137. 10ن010111 CCC1110

139. 20100001111 CC110

141. $1001011100 C 01110$

143. ccolcillo1ccillo

145. C10101111 cecello

147. 1C012000C1011110

14\%. C1111010100 COL10 1E1. CCu111101CC10110 153. $1111010 \operatorname{col} 1 \mathrm{CO} 10$ 155. $11100101116 \mathrm{COO} 10$ 157. 1000111100010110 155. 0001001111611010 161. CC1110111CCC1010 163. Cl1C0CC101111010
2. 1000010111100110

4. 0001110100111010

6. 0001100101111010

8. 0111010006111010

10. 0101111010000110

12. $111101000011001 \mathrm{C}$

14. 0001010011110110

16. 0001111001101010

18. 1110001011100010

20. 1100010001111010

22. 0011100010101110

24. 1111000011010010

26. 1110001110001010

28. $010111101100001 \mathrm{C}$

30. 0001100111101010

32. 1100011110100010

34. 1111000011000110

36. 1111000100010110

38. 1010011000011110

40. 1111000110100610

42. 1110100001110010

44. 1111001003010110

46. 0001110011101010

48. 0111101101000010

50. 0011000101011110

52. 1110111000010010

54. 0001011110010110

56. 0111100001010110

58. 0010100011101110

60. 0001101111010010

62. 1011001000011110

64. 0101011100001110

66. 0001011001011110

68. 0001111011001010

70. 1000111101000110

72. 1100001111001010

14. 1010011110000110

70. 0011110001011010

$7 \dot{~ i s . ~} 1000011110100110$

85. 0100001011011110

32. 1110111001000010

64. 1111001101000010

36. 1110000111001010

48. 1111011000010010

90.0011110110100010

72. $11110 \mathrm{C} 10 \mathrm{COC} 11010$

94. 1101 s0001 Ju11110

46. 1100001311110010

98. 1011110010000110

100. 0011116101100010

102. 1011110000100110

1U4. 0101110100001110

166. 0101100001111010

108. 0001001011101110

110. 0001111016011010

112. 1110000160111010

114. 0011011110100010

116. 0001001111010110

11\%. 0010101100011110

120. 101110 v001001110

122. 11111000010110010

124. U11luenolulol110

120. U010111141100010

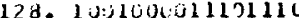

130. $100001 \div 110011110$

13ź. 1001116000101110

134. 1 0iov01110001110

136. 1 U100011110010110

138. 0111011100001010

140. 1000101100011110

142. 1000010111001110

144. 1111011000100010 146. 0001011010011110 148. 0010111100010110 150. 0101111060011010 152. 1100101111000010 154. 0010110111100010 150́. 11110001110001010 158. 1000011101001110 1t0. 1110000101110010 162. 0101000011011110 164. 1101000011110010
165. $001110111 \mathrm{Cl} 0010$ 167. $00111010001 \mathrm{C1110}$

169. 0101110111000010

171. 0001010111001110

173. $100111101 \mathrm{CCC} 0110$

175. Cc010011101C1110

177. 0001001116111010

179. C110100001111010

181. C101000011110110

183. $10001110111 \mathrm{CO} 10$

1Es. $11100100001 \mathrm{c11} 10$

187. C011110001010110

189. Co01011001111010

151. 0010111010001110

153. I0C1100001111010

195. $11110100110 c 0010$

157. $10111100 \mathrm{C} 1 \mathrm{CCC110}$

199. 1110011101000010

201. 1011110011060010

2C3. 111100010 C0.11010

2C5. $10001011110 \mathrm{CO110}$

2C7. 1000010011110110

2c9. 00111101010C0110

211. $000110101 \mathrm{Cc} 11110$

213. $11101001000 C 1110$

215. 011101C10CCC1110

217. 0001101011110010

219. 1101000111100010

221. $011100001 \mathrm{Cl} 11010$

223. $000111101 \mathrm{Cl} 1001 \mathrm{c}$

225. 1111C001011C0010

227. $0001101111 \mathrm{CC1010}$

229. I1CC10000ICH1110

231. $\operatorname{Col} 01011110 \operatorname{col} 10$

233. C0011101010C1110

235. CC1010111CCC1110

237. 1000011016011110

239. 1011100001110010

241. 1111001100001010

243. C0111000111C1010

245. 111101000CICO110

247. 111010001 COC1110

249. 11101000111 c0010

251- 0001011011110010

253. $10001000111 \mathrm{C1110}$

255. 1000011101110010

257. C1111000011C1010

259. 1011100111000010

261. $10100001110 C 1110$

263. $00101111010 C 0110$

265. 1100001001011110

267. 0001110111001010

269. $101111000 \mathrm{C} 110010$

271. C101101000011110

273. C110000101011110

275. $00111101100 \mathrm{C} 1010$

277. 1001000011110110

279. 1000110111100010

281. 0111100001011010

283. 0110101006011110

2E5. 110100010 Co11110

287. C1011011110C0010

289. 111000010C1C1110

291. 0101100001011110

253. $C 010110001011110$

255. 1C110001111C0010

257. $111001110 C O C 1010$

259. $00011101001 C 1110$

3C1. CCO111101ClCOL10

3C3. CLIL10100CO10110

3C5. 1000110001111010

367. C1100001111C1010

3C9. 10100011110 C0110

311. 1100101000011110

313. 1101111006010010

315. CC100010111C1110

317. 1100100001111010

319. 1111000010011010

231. C100001111C 1C110

323. Cl11101C11C CCOLO

166. 0001011101110010

168. 0010100011110110

170. 1110100111000010

172. 0101011000011110

174. 0001111001011010

176. 1001110100001110

178. 0001110010111010

180. 1001110000111010

182. 0110100001011110

184. 0010111000101110

186. 0011110100011010

188. 1010000110011110

190. 0001011100101110

192. 1000110001011110

194. 01111 C1000011010

196. 1011100100001110

198. 1110010000111010

200. 1111000101000110

202. 0001101001111010

204. 0001001101111010

206. 1100111100001010

208. 1000011110110010

210. 0101110000101110

212. 1011000100011110

214. 0001010110011110

216. 0001001011011110

218. 1011100010001110

220. 0111000011101010

222. 1011000011110010

224. 1001101111000010

226. 1000100011011110

228. 0001111001010110

$23 \mathrm{c}$. 0010001111011010

232. 1101001111000010

234. 0010001110101110

236. 1011110001100010

238. 1000011001011110

240. 1100111101000010

242. 0010110100011110

244. 1100010100011110

246. 1110100001001110

248. 0010001011011110

250. 1101111001000010

252. 0110101111000010 


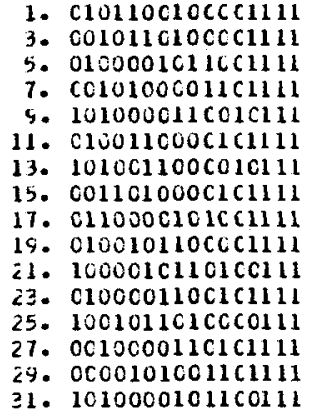

2. 1000010011010111

4. 0101100001001111

6. 1001101000010111

8. 1011000010100111

10. 1001000011010111

12. 0000101101001111

14. 1010110000100111

16. 1000011010010111

18. 0000110100101111

20. 0110010100001111

22. 1011001010000111

24. 1010110010000111

26. 1010010110000111

28. 1001010000110111

30. 100001010011011

32. 0000100110101111

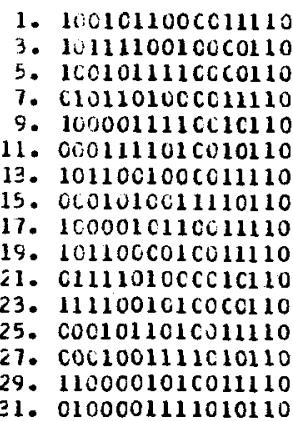

2. 0101111010000110

4. 1011110000100110

6. 1111000010100110

8. 0101000011110110

10. 0101000011011110

12. 1001100001011110

14. 0001010011011110

16. 0110100001011110

18. 1000010111100110

20. 0001001101011110

22. 0001011110100110

24. 1100101000011110

26. 1000011001011110

28. 0100001101011110

30. 0001101001011110

32. 1001111000010110

TERMINAL EVENTS OF FRECEDING SEQUENCE : 100

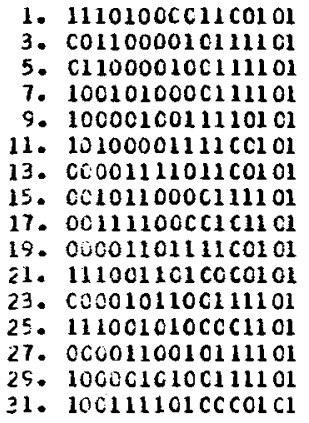

2. 1110000100110101

4. 1110000101001101

6. 0011110110000101

3. 0010111100001101

10. 1110100110000101

12. 1001000011110101

14. 0111100001001101

16. 1110010000110101

13. 0011011110000101

2C. 0110010000111101

22. 0111100100001101

24. 0000101111001101

26. 0000111100101101

23. 1000011110100101

30. 1110000110100101

32. 1010011110000101
1. 0011110101160100

3. $100001101 \mathrm{C} 111100$

5. $11 \times 101111 \mathrm{CCCClCO}$

7. $10100001111 \mathrm{C11} 00$

9.1100001011110100

11. $110111100 C C 10100$

13. 0011110110010100

15. 1101000016111100

17. CC1011010C111100

15. $111100001 \mathrm{Cl} 101 \mathrm{CO}$

21. 111101011C

23. CO1111010C1C1100

25. $001111001 C 110100$

27. $100001111 \mathrm{ClC1100}$

29. $\mathrm{CO} 1001111 \mathrm{C1C1100}$

z1. Col10101111COLCO
2. 1011000011110100
4. 0010100110111100
6. 0011010010111100
8. 0010111100110100
10.0010011010111100
12. 1111011000010100
14. 0010111101001100
16. 1011110000110100
18.0011011110010100
20.0010100111101100
22.1111010000101100
24.1010000110111100
26.1011110100001100
28.1011010000111100
30.0011001011110100
32.0010110011110100 of the r-tuple(s) begun in the block preceding it and, therefore, the r-tuple distribution for a trial block is contingent upon the terminal event(s) of the preceding block. Consequently, all sequences are listed in terms of those appropriate following specific terminal events of the preceding sequence. For 2-tuple balance, it is necessary to take into account only the single terminal event of the preceding block, whereas the final two events must be considered for 3-tuple balance, etc.

of course, in some experimental contexts in which blocks of trials are separated by long periods of time, independence between trial blocks may be postulated and series may be chosen without regard to preceding blocks. In order to obtain all possible r-tuples in such situations, it is necessary to lengthen each basic block by preceding it with appropriate events chosen from the terminal events section.

(3) Directly related to the latter point of (2) above, if all trial blocks in an experiment are to be submitted to sequential analysis, the first complete block of trials must be preceded by an appropriate set of events chosen from the terminal events section. For example, if an experiment involves five interrelated trial blocks of length 8 balanced for 3-tuple occurrences, and if the first block is to be submitted to sequential analysis, the experiment will consist of 42 trials: the initial two trials of the experiment (which are not analyzed but provide the starting points for 3-tuples terminating in the first complete block) and five blocks of 8 trials each.

(4) Since each sequence, along with its appropriate terminal events, has a complement or mirror image (e.g., 1011 is the mirror image of 0100 ), only half of the possible sequences are actually presented in these tables. Omissions were made on the basis of the terminal events of preceding sequence classifications with only those terminal events sections starting with 1 included in the tables.

(5) If an experiment involves a succession of interrelated as opposed to independent blocks, once it is initiated in a given terminal events section it will remain in that section for its duration, i.e., the terminal events for each block are the same across all blocks. This, of course, results in differences in the average placement values of the various r-tuples that cannot be eliminated on a within-Ss basis, as well as trial-by-trial probabilities for each event class which deviate from .5 within each terminal events section. As can be seen in Table 7, the relationships between these probabilities and trials are quite complex and, hopefully, will not be reflected in behavioral fluctuations in most experimental contexts. Any standard counterbalancing strategy in which equal numbers of Ss are given schedules from all possible terminal events sections, including those which are complementary to the sections appearing in the present tables, will result in comparable placement values for r-tuples and trial-by-trial probability values of .5 when averaged across Ss. Similar counterbalancing techniques, of course, would have to be employed with any method of schedule generation if comparable placement values were achieved for each trial block.

(6) Within a given terminal events section, the ordering of the sequences was determined by a computer program which assigned a random number to each sequence. The numbers appearing to the left of the sequences represent the ordinal 
Table 7

Probabilities of a 1 Occurrence for Each Trial Related to Sequence and Subsequence Lengths and Terminal Events of Preceding Block*

\begin{tabular}{|c|c|c|c|c|c|c|c|c|c|c|c|c|c|c|c|c|c|c|c|}
\hline \multirow[b]{2}{*}{ Table } & \multirow{2}{*}{$\begin{array}{c}\text { Sequence } \\
\text { Length }\end{array}$} & \multirow{2}{*}{$\begin{array}{l}\text { Sub- } \\
\text { sequence } \\
\text { Length }\end{array}$} & \multirow{2}{*}{$\begin{array}{c}\text { Terminal } \\
\text { Events }\end{array}$} & \multicolumn{16}{|c|}{ Trial } \\
\hline & & & & 1 & 2 & 3 & 4 & 5 & 6 & 7 & 8 & 9 & 10 & 11 & 12 & 13 & 14 & 15 & 16 \\
\hline 1 & 4 & 2 & 1 & 0.50 & 0.00 & 0.50 & 1.00 & & & & & & & & & & & & \\
\hline 2 & 8 & 2 & 1 & 0.50 & 0.33 & 0.39 & 0.56 & 0.39 & 0.33 & 0.50 & 1.00 & & & & & & & & \\
\hline \multirow[t]{2}{*}{3} & 8 & 3 & 11 & 0.50 & 0.25 & 0.25 & 0.25 & 0.25 & 0.50 & 1.00 & 1.00 & & & & & & & & \\
\hline & & & 10 & 0.50 & 0.25 & 0.75 & 0.25 & 0.75 & 0.50 & 1.00 & 0.00 & & & & & & & & \\
\hline 4 & 12 & 2 & 1 & 0.50 & 0.40 & 0.44 & 0.48 & 0.47 & 0.42 & 0.47 & 0.48 & 0.44 & 0.40 & 0.50 & 1.00 & & & & \\
\hline \multirow[t]{2}{*}{5} & 16 & 3 & 11 & 0.50 & 0.42 & 0.33 & 0.42 & 0.46 & 0.42 & 0.45 & 0.45 & 0.42 & 0.46 & 0.42 & 0.33 & 0.42 & 0.50 & 1.00 & 1.00 \\
\hline & & & 10 & 0.50 & 0.42 & 0.50 & 0.58 & 0.46 & 0.50 & 0.53 & 0.47 & 0.50 & 0.54 & 0.42 & 0.50 & 0.58 & 0.50 & 1.00 & 0.00 \\
\hline \multirow[t]{4}{*}{6} & 16 & 4 & 111 & 0.50 & 0.25 & 0.44 & 0.28 & 0.41 & 0.47 & 0.31 & 0.47 & 0.41 & 0.28 & 0.44 & 0.25 & 0.50 & 1.00 & 1.00 & 1.00 \\
\hline & & & 110 & 0.50 & 0.38 & 0.25 & 0.72 & 0.34 & 0.47 & 0.62 & 0.28 & 0.59 & 0.41 & 0.19 & 0.75 & 0.50 & 1.00 & 1.00 & 0.00 \\
\hline & & & 101 & 0.50 & 0.38 & 0.62 & 0.28 & 0.47 & 0.59 & 0.31 & 0.59 & 0.47 & 0.28 & 0.62 & 0.38 & 0.50 & 1.00 & 0.00 & 1.00 \\
\hline & & & 100 & 0.50 & 0.25 & 0.81 & 0.59 & 0.41 & 0.72 & 0.38 & 0.53 & 0.66 & 0.28 & 0.75 & 0.63 & 0.50 & 1.00 & 0.00 & 0.00 \\
\hline
\end{tabular}

*In each case the use of all terminal events sections, including complements to those tabled, will result in trial-by-trial probabilities of .5 when averaged across Ss.

positions which resulted and can be used as the basis for selection by means of a lottery technique or a table of random numbers.

\section{DISCUSSION}

Use of the present tables represents one of several approaches to developing schedules for psychological experiments in which two-class events are presented. The unique properties which these schedules possess are that equal numbers of the possible overlapping subsequences, or r-tuples, occur within each trial block. Various other methods of schedule generation will result in such characteristics with varying degrees of success.

A method used frequently is the random generation of events within restrictions of equal occurrences of the two classes of events within a trial block of specified length. Since the present sequences represent subsets of such series, it is obvious that r-tuple balance will occur with low probabilities when trial blocks are generated in this manner. For example, for 2-tuple balance probability values of .333, $.257, .219$, and .190 are obtained for trial-block lengths of $4,8,12$, and 16, respectively. With regard to 3-tuple balance, values of .057 and .025 are obtained for trial-block lengths of 8 and 16 , with a value of .002 occurring for 4-tuple balance of randomly generated 16-trial blocks having equal occurrences of the two event classes.

While a random method of schedule generation results in low probabilities of r-tuple balance, use of the Gellermann series will never result in equal r-tuple representation, due to the fact that each series contains 10 events. As discussed earlier, a basic requirement for such balance is that a trial block must be some multiple of a basic $2^{\mathrm{r}}$ unit, where $r$ represents the tuple length

A method that will result in trial blocks balanced for 2-tuple occurrence is that involving an equal number of runs of lengths 1,2 , and 3 of each class of event. However, while thes method will ensure balance for trial $\mathrm{n}-1$, or 2-tuple occurrence, when transitions occur at the junction of two blocks, these schedules are biased such that all overlapping subsequences having opposite first and third events (i.e., 110, 100, 001, and 011) necessarily occur with greater frequency than do those 3-tuples that have identical first and third events $(101,111,010$, and 000 ). This means that the probability that trials $n$ and $n-2$ are of the same class is less than .5. While some schedules in the present tables contain a similar bias (i.e., balancing for r-tuples means that only the effects of the $r-1$ trials preceding the nth trial can be assessed without possible bias), it is possible to select schedules from these tables which take into account the specific $\mathbf{n}-2$ bias of the type under discussion.

There are two ways in which sequential bias of the type currently under discussion might be important in a given experimental context. In the first place, the possibility exists that the $S$ may learn to respond congruent to this pattern during the course of the experiment, in which case measures of differential responding, etc., might be influenced. Second, it may be desirable and/or necessary to take into account possible reinforcement effects of preceding trials on trial $\mathrm{n}$. Indeed, existing data in differential eyelid conditioning with human Ss suggests that such effects (specifically for $\mathrm{n}-2$ effects) might be pronounced (Vandament, 1970). Therefore, the possibility exists that use of schedules biased in this regard as a result of balancing for run length could lead to lower apparent levels of differentiation than could use of schedules not characterized by this bias.

The relation of run length to r-tuple balancing can be more fully appreciated when one considers the restrictions concerning runs which necessarily accompany r-tuple balance. Such restrictions lead to some bias concerning run lengths in the present tables. First, the maximum run length of a given event within a trial block is related to the length of the r-tuple for which balancing has occurred and to the length of the trial block. If the basic $2^{r}$ unit is utilized as the trial block, the maximum run length within a block is necessarily the length of the defined r-tuple. Note, however, that run lengths greater than $r$ can occur at the junction of two trial blocks within the larger context of a total experiment. If some multiple of the basic $2^{r}$ unit is employed as a trial-block length, then the maximum run length within a trial block can be increased by 1 for each multiple greater than 1. For example, in Table 1 2-tuple frequency in a four-trial block is balanced, and runs of length 2 are the maximum observed within a trial block. In Table 2 trial blocks of length 8 are balanced for 2-tuples, and a maximum run length of three can occur within a trial block.

Furthermore, the probabilities associated with runs of certain lengths less than the maximum are also relatively low in some of the schedules due to the relationships between r-tuple patterns and run lengths and to the lack of independence which necessarily exists between the ordering of r-tuples. For example, a run of length 2 occurs only when the 3-tuples, 100 and 001 , are adjacent and thus form the event sequence 1001. For that case in which each 3-tuple occurs once (i.e., the sequence of length 8 balanced for 3-tuple frequency, Table 3), the 3-tuple 000 is necessarily bounded by 100 and/or 001 , and thus the possibility of a run of length 2 within the trial block is precluded. Similarly, runs of length 3 do not occur within the trial block when 16-trial sequences are balanced for 4-tuple frequency and maximum run length is 4 . Such runs of intermediate length can appear occasionally at the junction of two trial blocks within the context of a multiblock experiment, but they are, of 
course, characterized by relatively low probabilities of occurrence.

The significance of these sources of bias related to run length will necessarily be determined empirically for various experimental contexts. It should be noted, however, that (1) restrictions on maximum run length are also characteristic to some extent of all alternative methods of schedule generation described previously, and (2) any schedule that is balanced for run-length frequency will also contain some bias with regard to r-tuple occurrence which is more restrictive than the similar, but in some cases avoidable, bias which is inherent in the present schedules.

Finally, consideration should be given to alternation behavior, although the present sequences were not developed to deal specifically with such problems and not every sequence listed will yield a chance score of .5 when the $S$ displays a single- or double-alternation pattern. The authors are not aware of any published sets of events in which each series will result in a .5 "correct" probability associated with either type of alternation behavior. In the Gellermann series, a single-alternation pattern of behavior will result in a "correct" proportion of either .6 or .4 , depending upon the nature of the initial response. Double-alternation behavior can result in "correct" proportions of $.9, .7, .5$, .3 , or .1 for the Gellermann series. Summed across all possible sequences, "correct" probabilities of .5 are characteristic of both the Gellermann and the present sequences. Since a few of the present sequences have been found to yield "correct" scores of .5 for both single- and double-alternation patterns, the authors are presently attempting to survey the present tables for sets of sequences having the desired properties.

\section{CONCLUSIONS}

The preceding assessment of existing methods for generating schedules of two-class events indicates that not all desired features can be assured in any given schedule, e.g., r-tuple balance and equal representations of various run lengths cannot occur in the same schedule, and a random method of generation will rarely result in r-tuple balance. Therefore, the $E$ must determine in each case which properties are expendable and which are essential. However, it would appear crucial that the nature of schedule generation be reported in detail and desirable that standard schedule properties exist for some experimental areas in order to facilitate comparisons of results. In those contexts in which behavior has been demonstrated to be a function of immediately preceding reinforcements and nonreinforcements, the present schedules would appear to possess characteristics relevant to such standards.

\section{REFERENCES}

BRADLEY, J. P. Complete counterbalancing of immediate sequential effects in a Latin Square design. Journal of the American Statistical Association, 1958, 53, 525-528.

COCHRAN, W. G., \& COX, G. M. Experimental designs. (2nd ed.) New York: Wiley, 1957.

FREY, P. W. Differential rabbit eyelid conditioning as a function of age, interstimulus interval, and cue similarity. Journal of Experimental Psychology, 1969, 81, 326-333.

GELLERMANN, L. W. Chance orders of alternating stimuli in visual discrimination experiments. Journal of Genetic Psychology, 1933, 42, 206-208.

GRANT, D. A. The Latin Square principle in the design and analysis of psychological experiments. Psychological Bulletin, 1948, 45, 427-442.

MOORE, J. N. Differential eyelid conditioning as a function of the frequency and intensity of auditory conditioned stimuli. Joumal of Experimental Psychology, 1964, 68, 250-259.

PATTERSON, H. D. The construction of balanced designs for experiments involving sequences of treatments. Biometrika, 1952, 39, 32-48.

VANDAMENT, W. E. Effects of CS-UCS interval, distance separating the CSs, and sequential context in differential eyelid conditioning. Unpublished manuscript, State University of New York at Binghamton, 1970.

\section{NOTES}

1. This project was supported in part by National Science Foundation Grant GB-4492. Appreciation is expressed to Norman $\mathrm{H}$. Anderson and George W. Hiller III for their helpful comments. The tables were generated at the Computer Center, State University of New York at Binghamton.

2. Presently at Massachusetts Institute of Technology. 